\title{
Rubrofusarina, um policetídeo natural inibidor da topoisomerase II- $\alpha$ humana
}

\author{
Alexsandro Branco, ${ }^{* 1}$ Angelo C. Pinto, ${ }^{2}$ Raimundo Braz-Filho, ${ }^{3}$ Edson F. Silva, ${ }^{4}$ \\ Noema F. Grynberg, ${ }^{4}$ Aurea Echevarria ${ }^{4}$ \\ ${ }^{1}$ Departamento de Saúde, Universidade Estadual de Feira de Santana, Av. Universitária s/n, BR 116 - Km 03, \\ Campus Universitário, 44031-460 Feira de Santana-BA, Brasil,

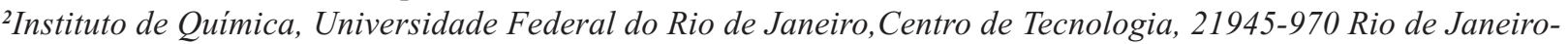 \\ RJ, Brasil, \\ ${ }^{3}$ Setor de Química de Produtos Naturais, LCQUI-CCT, Universidade Estadual do Norte Fluminense, 28015-620 \\ Campos dos Goitacazes-RJ, Brasil, \\ ${ }^{4}$ Departamento de Química, Instituto de Ciências Exatas, Universidade Federal Rural do Rio de Janeiro, \\ 23851-970 Seropédica-RJ, Brasil
}

\begin{abstract}
RESUMO: Este trabalho descreve o efeito inibitório de rubrofusarina (5,6-diidroxi-8-metoxi-2metilbenzo[g]cromen-4-ona, 1) sobre a enzima DNA topoisomerase II- $\alpha$ humana. Rubrofusarina mostrou total inibição da enzima topisomerase II- $\alpha$ na concentração de $120 \mu \mathrm{M}$. Este resultado é semelhante ao observado com etoposida, utilizada como controle positivo. Para a realização deste teste, rubrofusarina foi isolada de Senna macranthera var. nervosa (Voguel) Irwin \& Barnebyem e caracterizada por métodos espectroscópicos, incluindo RMN 2D, do produto natural bem como de seu derivado monoacetilado.
\end{abstract}

Unitermos: Rubrofusasina, topoisomerase, Senna macranthera, Fabaceae.

\begin{abstract}
Rubrofusarin, a natural polyketide as new human topoisomerase II- $\alpha$ inhibitor". This work describes the inhibitory effect of rubrofusarin (5,6-dihydroxy-8-methoxy2 -methylbenzo[g]cromen-4-one, 1) against human DNA topoisomerase II- $\alpha$. The results for relaxation assays showed total inhibition of topisomerase II- $\alpha$ by rubrofusarin at $120 \mu \mathrm{M}$. This result is comparable to the one observed with etoposide as positive control. For this study, rubrofusarin was isolated from Senna macranthera var. nervosa (Voguel) Irwin \& Barnebyem and characterized by spectral data, including 2D NMR, as well as its acetylated derivative.
\end{abstract}

Keywords: Rubrofusarin, topoisomerase, Senna macranthera, Fabaceae.

\section{INTRODUÇ̃̃O}

As topoisomerases são enzimas nucleares que controlam e modificam o estado topológico do DNA. Estas enzimas nucleares são classificadas em tipo I e II, de acordo com seus mecanismos e propriedades físicas, em células de mamíferos. A topoisomerase II (Topo II), dímero de tamanho aproximado de $170 \mathrm{kDa}$ e existente nas isoformas $\alpha$ ou $\beta$, é responsável pela quebra da fita dupla de DNA levando a eventos como liberação da fita de DNA enovelada, transcrição, condensação dos cromossomos e recombinação (Sampaio et al., 2006; Hande, 2006). As topoisomerases, durante a proliferação celular, participam da manutenção e replicação do DNA. Quando estas funções são desativadas, as células ficam vulneráveis. Além disso, em tecidos tumorais a expressão das DNA-Topo I e II é maior do que nas células de metabolismo normal (Kellner et al., 2000). Assim, as topoisomerases são alvos importantes no desenvolvimento de novas drogas, principalmente para as que apresentam atividade anticâncer e antiviral (De Vita Jr et al., 1997).

$\mathrm{Na}$ medicina destacam-se os compostos das classes das antraciclinas e das epipodofilotoxinas como potentes inibidores da topoisomerase II. Estes compostos agem inibindo a religação do DNA e induzem a ligação de proteínas em suas quebras, e constituemse como parte integrante em quimioterapia de primeira linha para uma grande variedade de tumores sólidos e hematológicos (Sampaio et al., 2006). O etoposídeo, derivado semi-sintético da lignana podofilotoxina, apresenta papel importante no tratamento clínico como potente agente quimioterapêutico para uma variedade de tumores, incluindo carcinomas, câncer testicular e linfomas (Zhang et al., 1992). Na tentativa de descobrir novos inibidores da topoisomerase, diversas classes de produtos naturais têm sido testadas e descritas na literatura nos últimos anos. Dentre estas classes, 
podemos citar os flavonóides (Constantinou et al., 1995; Shi et al., 1995) e biflavonóides (Grynberg et al., 2002), diterpenos (Maciel et al., 2007) e triterpenóides (Wada et al., 1998), estilbenóides (Yamada et al., 2006), alcalóides (Woo et al., 1997; Prescott et al., 2007), naftodiantronas (Peebles et al., 2001), naftoquinonas (Ting et al., 2003; Phyta et al., 1998; Esteves-Souza et al., 2007) e binaftoquinonas (Cunha et al., 2006), derivados do núcleo cromano (Ishar et al., 2006), entre outras.

Rubrofusarina (Figura 1), pertencente a classe das nafto- $\gamma$-pironas, é um composto natural comumente encontrado em fungos e plantas do gênero Fusarium (Leeper \& Staunton, 1984; Mock \& Robbers, 1969) e Senna (Graham et al., 2004; Barbosa et al., 2004), respectivamente. Este composto foi descrito pela primeira vez em 1937 por Ashley e colaboradores como um componente do metabolismo secundário do fungo Fusarium culmorum, entretanto sua estrutura somente estabelecida em 1962, através de derivatizações químicas e análises espectrais utilizadas na época (Leeper \& Staunton, 1984). No ano seguinte, foi descrita a síntese da dimetoxirubrofusarina a partir do cloreto do ácido 3,5-dimetoxi-benzóico (Shibata et al., 1963) e, em outro trabalho, a síntese biomimética de rubrofusarina serviu como modelo para reações de ciclisarção catalizada pela enzima policetídeo sintetase (Abell et al., 1986). Dentre as atividades biológicas descritas para rubrofusarina destacam as propriedades quelantes (Pereira et al., 1995), inibidora da enzima xantina oxidase e atividade antimicrobial (Song et al., 2004), atividade herbicida (Mata et al., 2003), ação estrogênica (El-Halawany et al., 2007).

Rhee et al. (2007) descrevem que um dos principais requisitos estruturais para a inibição da Topo II é a presença de um cromóforo-planar contendo de dois a quatro anéis aromáticos condensados. Substâncias com este tipo de cromóforo podem intercalar com o DNA, causando bloqueio ou erros na leitura enzimática durante o processo de replicação. Baseado neste requisito, a rubrofusarina (1) nos chamou atenção por apresentar o núcleo naftopirona, uma vez que esta característica estrutural pode conferir a este policetídeo capacidade inibitória da enzima topoisomerase.

Neste trabalho descrevemos o efeito inibitório de rubrofusarina (1) frente à enzima DNA topoisomerase II- $\alpha$ humana, usando como controle positivo a etoposida (3), derivado semi-sintético da lignana podofilotoxina (4), que apresenta papel importante no tratamento clínico como potente agente quimioterapêutico. Para a realização deste teste, rubrofusarina foi isolada das cascas do caule de $S$. macranthera e a sua estrutura identificada com base na análise dos dados espectrais (IV, EM e RMN 1D e 2D) do produto natural e de seu derivado monoacetilado, bem como pela comparação com dados da literatura.

\section{MATERIAL E MÉTODOS}

\section{Procedimentos gerais}

O ponto de fusão foi determinado em bloco de Koffler e não foi corrigido. O espectro de absorção na região de infravermelho foi registrado em espectrômetro Perkin-Elmer (modelo 1420), utilizando filme em pastilha comprimida de $\mathrm{KBr}$. Os espectros de RMN 1D e 2D foram obtidos em aparelho Bruker $200\left({ }^{1} \mathrm{H}: 200 \mathrm{MHz} ;{ }^{13} \mathrm{C}\right.$ : $50 \mathrm{MHz}$ ) e Bruker AC-300 $\left({ }^{1} \mathrm{H}: 300 \mathrm{MHz} ;{ }^{13} \mathrm{C}\right.$ : $\left.75 \mathrm{MHz}\right)$. Os deslocamentos químicos foram medidos em valores adimensionais $\delta$ (ppm) e as constantes de acoplamento (J) medidas em Hertz (Hz). Foram utilizados $\mathrm{CDCl}_{3}$ como solvente e tetrametilsilano como referência interna. $\mathrm{O}$ espectro de massas foi obtido por impacto de eletrônico $(70 \mathrm{eV}) \mathrm{em}$ espectrômetro de massas VG 7070. Para cromatografia em camada delgada analítica (C.C.D.) foram utilizadas cromatoplacas em folhas de alumínio contendo sílica gel de fabricação Merck $\left(\mathrm{PF}_{254}\right)$ e reveladas sob lâmpada de ultravioleta (comprimento de onda de 254 e $365 \mathrm{~nm}$ ).

\section{Material vegetal}

As cascas do caule de um espécime de Senna macranthera var. nervosa (Vogel) Irwin \& Barnebyem foram coletadas no Bairro de Barão Geraldo na cidade Campinas, estado de São Paulo, em novembro de 2000. A identificação botânica foi realizada pela bióloga Msc. Márcia Campos, do Jardim Botânico do Rio de Janeiro.

\section{Obtenção de rubrofusarina (1)}

As cascas $(500 \mathrm{~g})$ de $S$. macranthera foram secadas em estufa a temperatura aproximada de $60{ }^{\circ} \mathrm{C}$. Depois de secas e desfiadas manualmente, este material foi deixado imerso em hexano durante 7 dias. Durante o processo de evaporação do solvente, à pressão reduzida e temperatura controlada em evaporador rotatório, observou-se na parte interna do balão a formação de um precipitado de coloração avermelhada. Após a evaporação de parte do solvente $(80 \%)$, a solução extrativa em hexano foi deixada em repouso. Após 24 horas, observou-se a deposição de material de coloração avermelhada. O sobrenadante foi separado com auxilio de uma pipeta Pasteur e concentrado à pressão reduzida. Obteve-se 45,0 g de extrato. O material sólido de coloração avermelhada foi recristalizado com uma mistura de acetona e metanol $(1: 1)$, e forneceu a rubrofusarina $\mathbf{1}(2,1 \mathrm{~g})$.

\section{Rubrofusarina (1)}

Sólido alaranjado; Pf $212-214{ }^{\circ} \mathrm{C}$; IV $v_{\max }^{\mathrm{KBr}} \mathrm{cm}^{-1}$ : $3388(\mathrm{OH}), 1659(\mathrm{C}=\mathrm{O}), 1623,1586$ (anel aromático); 
RMN de ${ }^{1} \mathrm{H}\left(300 \mathrm{MHz}, \mathrm{CDCl}_{3}\right)$ e ${ }^{13} \mathrm{C}\left(75 \mathrm{MHz}, \mathrm{CDCl}_{3}\right)$ : ver Tabela 1; EM: $272\left(\mathrm{M}^{+}, 100 \%\right), 243(\mathrm{M}-\mathrm{H}-\mathrm{CO}$ ou $\mathrm{M}-\mathrm{CO}-\mathrm{H}, 47 \%)$ e $229\left(\mathrm{M}-\mathrm{CH}_{3}-\mathrm{CO}\right.$ ou $\mathrm{M}-\mathrm{CO}-\mathrm{CH}_{3}, 15$ $\%)$.

\section{Acetilação de 1}

À rubrofusarina $(\mathbf{1}, 20,5 \mathrm{mg})$ foi adicionada $5 \mathrm{~mL}$ de anidrido acético e DMAP em quantidade catalítica. A mistura reacional foi agitada, sob refluxo, durante 6 horas. Em seguida, foi adicionada água e a solução foi extraída em funil de separação com hexano (2 x). A fase orgânica foi secada com sulfato de sódio e, após filtração, concentrada sob pressão reduzida. Após sucessivas recristalizações obteve-se o composto monoacetilado 2 (16 mg). RMN ${ }^{1} \mathrm{H}\left(200 \mathrm{MHz}, \mathrm{CDCl}_{3}\right)$ e RMN ${ }^{13} \mathrm{C} 50 \mathrm{MHz}, \mathrm{CDCl}_{3}$ ): ver Tabela 1 .

\section{Teste de inibição da DNA Topo II- $\alpha$}

O experimento foi realizado com 2 unidades de topoisomerase II- $\alpha$, para $0,125 \mathrm{mg}$ de kDNA encadeado (DNA do cinetoplasto), por incubação (37 ${ }^{\circ} \mathrm{C}$ por 30 min.) em tampão tris $50 \mathrm{mM} \mathrm{pH}=8,0$, de acordo com as instruções da TOPOGEN (Columbus, OH, USA), ATP $10 \mathrm{mM}$, na ausência e na presença de várias concentrações de $\mathbf{1}(0-250 \mu \mathrm{M})$ e da etoposida (3) usada como controle positivo (Chen \& Liu, 1994; Esteves-Souza et al., 2006).

Os resultados do ensaio foram analisados por eletroforese em gel de agarose $1 \%$, e posteriormente corados com brometo de etídio, sendo a revelação feita por irradiação em transiluminador de UV e imediatamente fotografada com máquina digital.

\section{RESULTADOS E DISCUSSÃO}

O extrato em hexano das cascas de $S$. macranthera forneceu um precipitado de coloração avermelhada identificado como rubrofusarina (1). O espectro na região de IV de $\mathbf{1}$ apresentou bandas de absorção de grupos hidroxila $\left(v_{\max } 3388 \mathrm{~cm}^{-1}\right)$, carbonila conjugada $\left(v_{\max } 1659 \mathrm{~cm}^{-1}\right)$ e anel aromático $\left(v_{\max } 1623\right.$ e $\left.1586 \mathrm{~cm}^{-1}\right)$. Através da análise do espectro de RMN de ${ }^{13} \mathrm{C}$-Pendant, deduziu-se o deslocamento químico e o número de hidrogênios para cada carbono de $\mathbf{1}$ (Tabela 1). O espectro de massas mostrou o íon molecular de $m / z 272\left(\mathrm{M}^{\bullet+}, \mathrm{C}_{15} \mathrm{H}_{12} \mathrm{O}_{5}\right)$, que juntamente com os dados obtidos pelo espectro de RMN de ${ }^{1} \mathrm{H}$, levaram a expansão da respectiva fórmula molecular para $[\mathrm{C}]_{8} \quad[\mathrm{C}=\mathrm{O}]$ $[\mathrm{CH}]_{4}\left[\mathrm{CH}_{3}\right]\left[\mathrm{OCH}_{3}\right][\mathrm{OH}]_{2}$. A presença de hidroxila quelatogênica em C-5 foi atribuída ao sinal singleto em $\delta_{\mathrm{H}} 15,95$. O espectro de RMN 2D ${ }^{1} \mathrm{Hx}^{1} \mathrm{H}$-COSY revelou apenas acoplamentos entre H-7 e H-9.

A correta atribuição dos átomos de hidrogênio e de carbono nos espectros de $\mathrm{RMN}$ de ${ }^{1} \mathrm{H}$ e de ${ }^{13} \mathrm{C}$ Pendant, respectivamente, foram acompanhados pelos

experimentos homonuclear 2D ${ }^{1} \mathrm{Hx}^{1} \mathrm{H}-\mathrm{NOESY} \mathrm{e}$ heteronuclear 2D ${ }^{1} \mathrm{Hx}^{13} \mathrm{C}-\mathrm{COSY}-{ }^{\mathrm{n}} \mathrm{J}_{\mathrm{CH}}(\mathrm{n}=1$, HMQC; $\mathrm{n}$ $=2$ e $3 \mathrm{HMBC}$ ) de 1 (Tabela 1). O espectro HMBC mostrou que os hidrogênios H-7, H-9 e H-10 estão correlacionados a longa distância $\left({ }^{3} \mathrm{~J}_{\mathrm{CH}}\right)$ com o carbono quaternário $\mathrm{C}-5 \mathrm{a}\left(\delta_{\mathrm{C}} 106,8\right)$, confirmando o sistema naftalênico da rubrofusarina (1). As demais correlações estão listadas na Tabela 1. O espectro homonuclear 2D ${ }^{1} \mathrm{Hx}{ }^{1} \mathrm{H}-\mathrm{NOESY}$ mostrou interações espaciais dos hidrogênios de metoxila em 3,90 (s) com os hidrogênios em H-7 $\left(\delta_{\mathrm{H}} 6,46 ; d, 2,2 \mathrm{~Hz}\right)$ e H-9 $\left(\delta_{\mathrm{H}} 6,56 ; d, 2,2 \mathrm{~Hz}\right)$ e de metila em 2,40 $(s)$ com o hidrogênio em H-3 $\left(\delta_{\mathrm{H}}\right.$ $6,01, s)$. Este espectro apresentou, também, sinal de interação de H-10 $\left(\delta_{\mathrm{H}} 6,95, s\right)$ com o hidrogênio H-9.

Acetilação de rubrofusarina produziu o produto monoacetilado 2. O grupamento acetila em C-6 foi confirmado pelos sinais de grupo acetoxílico em $\delta_{\mathrm{H}}$ $2,34(\mathrm{~s})$ e $\delta_{\mathrm{C}} 170,3(\mathrm{C}=\mathrm{O})$ e $20,9\left(\mathrm{CH}_{3}\right)$ presentes nos espectros de $\mathrm{RMN}$ de ${ }^{1} \mathrm{H}$ e de ${ }^{13} \mathrm{C}$, respectivamente. Os dados espectrais obtidos indicam que a hidroxila quelatogênica em C-5 $\left(\delta_{\mathrm{H}} 14,6\right)$ foi mantida. O espectro de RMN de ${ }^{1} \mathrm{H}$ de $\mathbf{2}$ quando comparado com $\mathbf{1}$ (Tabela 1) mostrou deslocamento paramagnético dos sinais em $\delta_{\mathrm{H}} \mathrm{H}-7\left(\delta_{\mathrm{H}} 6,71 ; d, 2,2 \mathrm{~Hz}\right), \mathrm{H}-9(6,85 ; d, 2,2 \mathrm{~Hz})$ e H-10 $\left(\delta_{\mathrm{H}} 7,06, s\right)$, como consequência do efeito de desproteção do grupo acetoxílico. Os espectros de RMN de ${ }^{13} \mathrm{C}\left(\mathrm{HBBD}\right.$ e DEPT $\operatorname{com} \theta=135^{\circ}$ e $\left.90^{\circ}\right)$ mostraram os sinais correspondentes aos dezessete carbonos de $\mathbf{2}$ e permitiram verificar as modificações esperadas nos deslocamentos químicos correspondentes aos átomos de carbono localizados em posição orto $\left[\mathrm{C}-5 \mathrm{a}: \Delta \delta_{\mathrm{C}}=109,7\right.$ (2) - 106,8 (1) $=2,9$ ppm; CH-7: $\Delta \delta_{\mathrm{C}}=110,5$ (2) - 100,7 (1) $=9,8$ ppm] e para $\left[\mathrm{CH}-9: \Delta \delta_{\mathrm{C}}=103,7\right.$ (2) - 98,2 (1) $=5,5 \mathrm{ppm}]$ demonstrando a redução de densidade eletrônica como consequência da significativa atenuação do efeito mesomérico do átomo de oxigênio sustentado pelo carbono C-6 após a acetilação. Tais modificações confirmaram que a monoacetilação envolveu somente a hidroxila localizada neste átomo de carbono (C-6).

Assim, o policetídeo rubrofusarina 1 isolado de Senna macranthera var. nervosa (Vogel) Irwin \& Barnebyem e seu derivado monoacetilado foram caracterizados como 5,6-diidroxi-8-metoxi2-metilbenzo[g]cromen-4-ona (1) e 5-O-acetil-6diidroxi-8-metoxi-2-metilbenzo[g]cromen-4-ona (2), respectivamente.

\section{Teste de inibição da enzima DNA Topo II- $\alpha$}

A presença do cromóforo de estrutura plana, em decorrência dos dois anéis aromáticos condensados, confere ao composto rubrofusarin (1) a possibilidade de interação com a enzima Topo II- $\alpha$. Devido a esta característica estrutural avaliou-se a possível ação de 1 sobre a DNA Topo II- $\alpha$ humana, no ensaio de relaxação do DNA super-helicoidizado (kDNA) (Chen \& Liu, 1994; Esteves-Souza et al., 2006). A Figura 2 
<smiles>COc1cc(OC)c2c(O)c3c(=O)cc([Al])oc3cc2c1</smiles>

$$
\begin{array}{ll} 
& \mathrm{R} \\
1 & \mathrm{H} \\
2 & \mathrm{AC}
\end{array}
$$<smiles>COc1cc([C@H]2c3cc4c(cc3[C@@H](O)C3COC(=O)[C@H]32)OCO4)cc(OC)c1O</smiles><smiles>C[C@@H]1OC[C@H](O)[C@H](O)[C@H](O)[C@H](O)O1</smiles><smiles>COc1cc([C@H]2c3cc4c(cc3C(O)C3COC(=O)[C@@H]32)OCO4)cc(OC)c1O</smiles>

3

4

Figura 1. Estrutura química de rubrofusarina (1), de seu derivado acetilado (2), da etoposida (3) e da podofilotoxina (4).

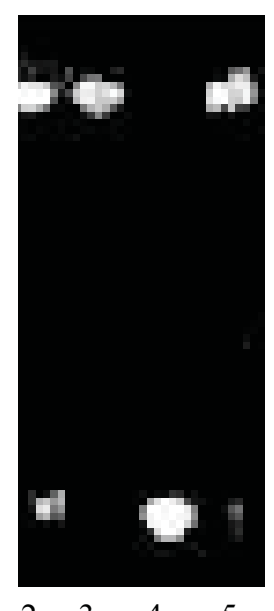

$2 \quad 3 \quad 4 \quad 5$

Figura 2. Eletroforese do ensaio com a rubrofusarina e a Topo II- $\alpha$ : $1.1(120 \mu \mathrm{M})+\mathrm{kDNA}+$ Topo II- $\alpha ; 2.1(60 \mu \mathrm{M})+\mathrm{kDNA}$ + Topo II- $\alpha$; $3.1(30 \mu \mathrm{M})+\mathrm{kDNA}+$ Topo II- $\alpha$; 4. kDNA puro; 5. kDNA + Topo II- $\alpha$.

mostra o resultado do ensaio de relaxação do kDNA, indicando que $\mathbf{1}$ apresentou total inibição da Topo II- $\alpha$ em concentração de $120 \mu \mathrm{M}$, ao se observar na linha 1 , a ausência da banda do kDNA relaxado; na concentração de $60 \mu \mathrm{M}$ evidenciou-se inibição parcial, já que as bandas do kDNA relaxado e super-helicoidizado são observadas (linha 2), enquanto que na concentração de $30 \mu \mathrm{M}$ não houve inibição (linha 3 ). Este resultado foi comparado com a etoposida, um inibidor bem conhecido e específico da Topo II- $\alpha$, que foi usado como controle positivo $(100 \mu \mathrm{M})$ apresentando perfil similar ao observado com a rubrofusarina (Figura 3). A Tabela 2

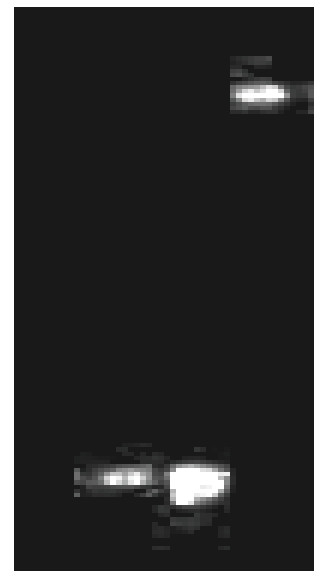

DNA relaxado

DNA super-helicoidizado

Figura 3. Eletroforese do ensaio com a etoposida (controle positivo) e a Topo II $\alpha$ : 1 . kDNA $(0,125 \mu \mathrm{g})+$ etoposida $(100$ $\mu \mathrm{M})+2 \mathrm{U}$ Topo II- $\alpha ; 2$. kDNA puro; 3 . kDNA $+2 \mathrm{U}$ Topo II- $\alpha$.

sumariza os resultados obtidos.

Rufrofusarina (1) apresentou atividade inibitória frente a DNA-Topo II- $\alpha$ humana, mostrando ser um bom protótipo para futuras investigações em direção a novos anti-virais e anti-tumorais.

\section{AGRADECIMENTOS}

Os autores agradecem Fundação de Amparo a Pesquisa do Estado do Rio de Janeiro (FAPERJ, Rio de Janeiro), Conselho Nacional de Desenvolvimento Científico e Tecnológico (CNPq), Fundação 
Tabela 1. Completa atribuição dos deslocamentos químicos $\left(\delta_{\mathrm{H}}\right.$ e $\left.\delta_{\mathrm{C}}\right)$ de ${ }^{1} \mathrm{H}(300 \mathrm{MHz})$ e de ${ }^{13} \mathrm{C}(75 \mathrm{MHz})$ de $\mathbf{1}$ e $2\left(\mathrm{CDCl}_{3}\right)$, incluindo os resultados dos experimentos heteronucleares $2 \mathrm{D} \mathrm{HMQC}\left({ }^{1} \mathrm{Hx}^{13} \mathrm{C}-\mathrm{COSY}-{ }^{1} \mathrm{~J}_{\mathrm{CH}}\right)$ e $\mathrm{HMBC}\left({ }^{1} \mathrm{Hx}^{13} \mathrm{C}-\mathrm{COSY}-\mathrm{J}_{\mathrm{CH}}, \mathrm{n}=2 \mathrm{e} 3\right)$ de 1 e comparação com dados de ${ }^{13} \mathrm{C}$ (em parênteses) descritos na literatura (Graham et al., 2004) para rubrofusarina (em piridina$\left.\mathrm{d}_{5}\right)^{*}$.

\begin{tabular}{|c|c|c|c|c|c|c|}
\hline & \multicolumn{4}{|c|}{1} & \multicolumn{2}{|r|}{2} \\
\hline & $\delta_{\mathrm{C}}$ & $\delta_{\mathrm{H}}$ & ${ }^{2} \mathbf{J}_{\mathrm{CH}}$ & ${ }^{3} \mathrm{~J}_{\mathrm{CH}}$ & $\delta_{\mathrm{C}}$ & $\delta_{\mathrm{H}}$ \\
\hline \multicolumn{7}{|l|}{$\mathbf{C}$} \\
\hline 2 & $169,1(169,7)$ & - & $\mathrm{H}-3 ; 3 \mathrm{H}-11$ & & 168,2 & - \\
\hline 4 & $184,3(184,2)$ & - & H-3 & $\mathrm{H}-10\left({ }^{4} \mathrm{~J}_{\mathrm{CH}}\right)$ & 184,9 & - \\
\hline 5 & $162,1(163,1)$ & - & HO-5 & HO-6 & 160,8 & - \\
\hline 6 & $158,8(159,5)$ & - & H-7; HO-6 & & 154,1 & - \\
\hline 8 & $163,0(163,3)$ & - & H-7; H-9 & $\mathrm{MeO}-8$ & 160,8 & - \\
\hline $4 a$ & $103,0(103,3)$ & - & & H-3; HO-5 & 103,8 & - \\
\hline $5 a$ & $106,8(107,4)$ & - & & $\begin{array}{l}\text { H-7; H-9; H-10; } \\
\text { HO-5; HO-6 }\end{array}$ & 109,7 & - \\
\hline $9 \mathrm{a}$ & $140,3(141,0)$ & - & & & 140,3 & - \\
\hline $10 \mathrm{a}$ & $152,6(153,0)$ & - & $\mathrm{H}-10$ & & 150,2 & - \\
\hline $\mathrm{Ac}$ & - & - & - & - & 170,3 & - \\
\hline \multicolumn{7}{|l|}{$\mathrm{CH}$} \\
\hline 3 & $106,7(106,5)$ & $6,01(s)$ & & $3 \mathrm{H}-15$ & 107,5 & $6,02(s)$ \\
\hline 7 & $100,7(101,1)$ & $\begin{array}{c}6,46 \\
(d, 2,2 \mathrm{~Hz})\end{array}$ & & & 110,5 & $6,71(d, 2,2)$ \\
\hline 9 & $98,2(98,5)$ & $\begin{array}{c}6,56 \\
(d, 2,2 \mathrm{~Hz})\end{array}$ & & H-7; H-10 & 103,7 & $6,85(d, 2,2)$ \\
\hline $\begin{array}{c}10 \\
\mathrm{CH}_{3}\end{array}$ & $101,4(101,4)$ & $6,95(\mathrm{~s})$ & & H-9 & 101,7 & $7,07(s)$ \\
\hline $\mathrm{MeO}-8$ & $56,6(55,4)$ & $3,90(s)$ & & & 55,7 & $3,92(s)$ \\
\hline 11 & $21,1(20,4)$ & $2,40(s)$ & & $\mathrm{H}-3$ & 21,4 & $2,41(s)$ \\
\hline Ac & - & - & - & & 20,9 & $2,34(s)$ \\
\hline $\mathrm{HO}-5$ & - & $15,95(s)$ & & & - & $14,5(s)$ \\
\hline HO-6 & - & $9,66(s)$ & & & - & - \\
\hline
\end{tabular}

* O número de átomos de hidrogênio ligado a cada átomo de carbono foi deduzido através da análise do espectro de $\mathrm{RMN}$ de ${ }^{13} \mathrm{C}$ Pendant. Espectros 2D ( ${ }^{1} \mathrm{Hx}{ }^{1} \mathrm{H}-\mathrm{COSY}$ e $\left.{ }^{1} \mathrm{Hx}{ }^{1} \mathrm{H}-\mathrm{NOEY}\right)$ também foram utilizados para estes assinalamentos.

Tabela 2. Efeito inibitório da rubrofusarina (1) sobre a Topo II- $\alpha$ utilizando o ensaio de relaxamento do kDNA.

\begin{tabular}{c|c|c}
\hline & $\begin{array}{c}\text { Concentração } \\
(\mu \mathrm{M})\end{array}$ & $\begin{array}{c}\text { Efeito } \\
\text { Inibitório }\end{array}$ \\
\hline $\mathbf{1}$ & 120 & ++++ \\
& 60 & ++ \\
Etoposida & 30 & n. i. ${ }^{\text {a }}$ \\
\hline
\end{tabular}

${ }^{a}$.n. i.: nenhuma inibição.

Universitária José Bonifácio (FUJB, UFRJ, Rio de Janeiro) e a PRONEX (4002) pelo apoio financeiro. Os autores também agradecem à botânica Márcia Campos, do Jardim Botânico do Rio do Rio de Janeiro, pela identificação da planta.

\section{REFERÊNCIAS}

Abell C, Bush BD, Staunton J 1986. Biomimetic syntheses of the polyketide fungal metabolites alternariol and rubrofusarin: models for cyclisation reactions catalysed by polyketide synthase enzymes. $J$ Chem Soc, Chem Commun 15-17.

Barbosa FG, Oliveira MCF, Braz-Filho R, Silveira ER 2004. Anthraquinones and naphthopyrones from Senna rugosa. Biochem Sys Ecol 32: 363-365.
Chen AY, Liu LF 1994. DNA topoisomerases: essential enzymes and lethal targets. Annu Rev Pharmacol Toxicol 34: 191-218.

Constantinou A, Mehta R, Runyan C, Rao K, Vaughan A, Moon R 1995. Flavonoids as DNA topoisomerase antagonists and poisons: structure-activity relationships. J Nat Prod 58: 217-225.

Cunha AS, Lima ELS, Pinto AC, Esteves-Souza A, Echevarria A, Câmara CA, Vargas MD, Torres JC 2006. Synthesis of novel naphthoquinone-spermidine conjugates and their effects on DNA-topoisomerases I and II- $\alpha . J$ Braz Chem Soc 17: 439-442.

De Vita Jr VT, Hellman S, Rosenberg SA 1997. Cancer Principles of practice of oncology. 5 ed. Philadelphia: Lippincott-Raven.

El-Halawany AM, Chung MH, Nakamura N, Ma C, Nishihara T, Hattori M 2007. Estrogenic and anti-estrogenic activities of Cassia tora phenolic constituents. Chem Pharm Bull 55: 1476-1482.

Esteves-Souza A, Pissinate K, Nascimento MG, Grynberg NF, Echevarria A 2006. Synthesis, cytotoxicity, and DNA-topoisomerase inhibitory activity of new asymmetric ureas and thioureas. Bioorg Med Chem 14: 492-499.

Esteves-Souza A, Figueiredo DV, Esteves A, Câmara CA, Vargas MD, Pinto AC, Echevarria A 2007. Cytotoxic and DNA-topoisomerase effects of lapachol amine derivatives and interactions with DNA. Braz J Med 
Biol Res 40: 1399-1402.

Graham JG, Zhang H, Pendland SL, Santarsiero BD, Mesecar AD, Cabieses F, Farnsworth NR 2004. Antimycobacterial naphthopyrones from Senna obliqua. J Nat Prod 67: 225-227.

Grynberg NF, Carvalho MG, Velandia JR, Oliveira MC, Moreira IC, Braz-Filho R, Echevarria A 2002. DNA topoisomerase inhibitors: biflavonoids from Ouratea species. Braz J Med Biol Res 35: 819-822.

Hande KR 2006. Topoisomerase II inhibitors. Update Canc Therap I: 3-15.

Ishar MPS, Singh G, Singh S, Sreenivasan KK, Singh G 2006. Design, synthesis, and avaluation of novel 6-chlro-/fluorochromone derivatives as potencial topoisomerase inhibitor anticancer agents. Bioorg Med Chem 16: 1366-1370.

Kellner U, Rudolph P, Parwaresch R 2000. Human DNAtopoisomerase - diagnostic and therapeutic implications for cancer. Onkologie 23: 424-430.

Leeper FJ, Staunton J 1984. The biosynthesis of rubrofurasin, a polyketide naphthopyrone from Fusarium culmorum: 13C NMR assignments and incorporation of 13C and $2 \mathrm{H}$ labeled acetates. $J$ Chem Soc, Perkin Trans I: 2919-2925.

Maciel MAM, Martins JR, Pinto AC, Kaiser CR, EstevesSouza A, Echevarria A 2007. Natural and semisynthetic clerodanes of Croton cajucara and their cytotoxic effects against Ehrlich carcinoma and human K562 leukemia cells. J Braz Chem Soc 18: 391-396.

Mata R, Gamboa A, Macias M, Santillán S, Ulloa M, González MC 2003. Effect of selected phytoxins from Guanomyces polythrix on the calmodulin-dependent activity of the enzymes cAMP phosphodiesterase and NAD-Kinase. J Agric Food Chem 51: 4559-4562.

Mock BH, Robbers JE 1969. Biosynthesis of rubrofusarin by Fusarium graminearum. J Pharm Sci 58: 15601562.

Peebles KA, Baker RK, Kurz EU, Schneider BJ, Kroll DJ 2001. Catalytic inhibition of human DNA topoisomerase II- $\alpha$ by hypericin, a naphthodianthrone from St John's wort (Hypericum perforatum). Biochem Pharmacol 62: 1059-1070.

Pereira EC, Demicheli C, Peixoto LC, Beraldo H, Tosi L 1995. A spectrometric study of the chelating properties of 6-galactosyl-rubrofusarin: $\mathrm{Mg}(\mathrm{II}), \mathrm{Al}(\mathrm{III}), \mathrm{Ni}(\mathrm{II})$ and $\mathrm{Cu}$ (II) complexes. J Braz Chem Soc 6: 381-386.

Phyta ZF, Li T, Papageorgiou VP, Mellidis AS, Assimopoulou NA, Pitsinos EM, Couladouros EA 1998. Inhibition of topoisomerase I by naphthoquinone derivates. Bioorg Med Chem 8: 3385-3390.

Prescott TAK, Sadler IH, Kiapranis R, Maciver SK 2007. Lunacridine from Lunasia amara is a DNA intercalating topoisomerase II inhibitor. $J$ Ethnophamacol 109: 289-294.

Rhee H, Park HJ, Lee SK, Lee C, Choo HP 2007. Synthesis, cytotoxicity, and DNA topoisomerase II inhibitory activity of benzofuroquinolinediones. Bioorg Med Chem 15: 1651-1658.

Sampaio Filho C, Bertoni VD, Sampaio C, Pimenta A, Brandão MA 2006. Agentes antineoplásicos. In: Penildon, S. (org.) Farmacologia. 7. ed. Rio de Janeiro: Guanabara Koogan, p. 1055-1070.
Shi Q, Chen K, Li L, Chang J, Autry C, Kozuka M, Konoshima T, Estes JR, Lin CM, Hamel E, McPhail AT, McPhail DR, Lee K 1995. Cytotoxic and antimitotic flavonols from Polanisia dodecandra. J Nat Prod 58: 475482.

Shibata S, Morishita E, Arima Y 1963. Synthesis of rubrofusarin dimethyl ether. J Chem Soc 11: 821-823.

Song YC, Li H, Ye YE, Shan YC, Yang YM, Tan RX 2004. Endophytic naphthopyrone metabolites are coinhibitors of xanthine oxidase, SW 1116 cell and some microbial growths. FEMS Microbiol Lett 241: 67-72.

Ting C, Hsu C, Hsu H, Su J, Chen T, Tarn W, Kuo Y, WhangPeng J, Liu LF, Hwang J 2003. Isodiospyrin as a novel human DNA topoisomerase I inhibitor. Biochem Pharmacol 66: 1981-1991.

Wada S, Tanaka R, Iida A, Matsunaga S 1998. In vitro inhibitory effects of DNA topoisomerase II by fernane-type triterpenoids isolated from a Euphorbia genus. Bioorg Med Chem Lett 8: 2829-2832.

Woo SH, Reynolds MC, Sun NJ, Cassady JM, Snapka RM 1997. Inhibition of topoisomerase II by liriodenine. Biochem Pharmacol 54: 467-473.

Yamada M, Hayashi K, Hayashi H, Ikeda S, Hoshino T, Tsutsui K, Tsutsui K, Linuma M, Nozaki H 2006. Stilbenoids of Kobresia nepalensis (Cyperaceae) exhibiting DNA topoisomerase II inhibition. Phytochemistry 67: 307-313.

Zhang Y, Shen Y, Wang Z 1992. Novel 4 $\beta$-arylamino derivatives of 3',4'-didemethoxy-3',4'-dioxo-4deoxypodophyllotoxin as potent inhibitors of human DNA topoisomerase II. J Nat Prod 55: 1100-1111. 